\title{
Design of An Intelligent Split-type Electricity Utilization Measurement and Control Terminal for Local Household Energy Management and Optimization
}

\author{
Baiming Xie ${ }^{1}$, Lefeng Cheng ${ }^{2 *}$, Chao Ding ${ }^{1}$, Shouyuan Shi ${ }^{2}$, Zhukui Tan ${ }^{1}$, Zhengjia Li $^{2}$, Bin Liu ${ }^{1}$, Jinxiu Hua ${ }^{2}$, Wenjing \\ Huang $^{2}$ \\ ${ }^{1}$ Electric Power Research Institute of Guizhou Power Grid Co., Ltd, Guizhou, Guiyang 550002, China \\ ${ }^{2}$ Suzhou Huatian Power Technology Co., Ltd, Jiangsu, Suzhou 215000, China
}

\begin{abstract}
It is of great significance to implement automatic demand response (ADR) in the energy Internet based on accurate measurement and control of electricity utilization devices using intelligent terminals. Current intelligent terminals lack flexibility and possess weak data collection and processing capabilities. On this basis, this paper developed an intelligent split-type electricity utilization measurement and control terminal for local household energy management and optimization. This intelligent terminal has capabilities of digital signal processing and infrared-based precision control, which is composed of two separate parts: the device body and the infrared controller. Among them, the device body includes DSP chip, electrical sampling circuit, ADC chip, WiFi module, ZigBee module, etc. The infrared controller contains single-chip microcomputer, ZigBee module, infrared encoding and transmit-receiving module, and lithium-ion battery. The device body is able to provide commands to the infrared controller according to the collected electricity utilization information, environmental information and comprehensive demand response requirements, thereby accurately adjusting the operating status of the loads, namely the electrical household appliances. Due to the split-type and rechargeable design, this intelligent terminal is able to adapt to a complex home environment, laying the hardware foundation for effective home energy management and optimization and facilitating household loads participating in demand response, especially automatic demand response.
\end{abstract}

\section{Introduction}

In recent years, with rapid development of cyberphysical systems (CPS), the concepts of energy Internet and smart energy [1-3], and the electricity market [4], demand response (DR) technology is an effective mean to solve the issues of renewable energy accommodating and real-time balance of power system supply and demand [5-7]. Among them, the automatic demand response (ADR) is an important embodiment of the power system and user sides to participate in the energy Internet $[1,8]$. With the improvement of people's living standards, the proportion of household electricity loads in the total social power load will continue to increase [9, 10]. The importance of household users' participation in demand response based on home energy management systems (HEMS) is increasingly prominent [10-13]. In addition, smart metering systems [14] and advanced metering systems [15] have been gradually applied to home users' energy management systems. The key to realizing the ADR of home-side loads is to develop smart electricity terminals that can achieve complete and accurate data collection of power consumption and flexible and effective load control functions $[16,17]$.
Regarding the related research of smart power terminals, at present, the intelligent controller developed in [18] has the functions of electrical metering, wireless communication, and remote control, but it can only perform on-off control and does not have load adjustment capability. An intelligent device design integrating infrared transceiver module and wireless communication module is proposed in [19], with remote control, communication networking and other functions, but only supports $38 \mathrm{kHz}$ carrier frequency infrared signal, and does not have the function of power consumption information collection. A smart power terminal is developed in [14] based on DSP chip. Thanks to the computing advantages of DSP, this device implements powerful data analysis functions, with remote control and timing control capabilities, but still simple on/off control. In [20], a type of demand response controller is designed with functions of power data acquisition and infrared remote control. However, due to the use of a single-chip microcomputer as the core chip, the computational processing capacity is limited, and it is difficult to support high-frequency sampling and data analysis functions. Its infrared control only supports the carrier with frequency of $38 \mathrm{kHz}$, no infrared encoding library is configured, the use of a slightly more complex,

\footnotetext{
* Corresponding author: Lefeng Cheng, chenglf_scut@163.com
} 
and does not consider the characteristics of infrared light linear propagation, did not mention the infrared transmitter head shape and layout.

From the above investigations, it obtains that most existing devices have the disadvantages of a simple control method and weak data acquisition and processing capabilities. The simple control method of non-stop and disconnection makes the adjustable electrical equipment such as air conditioners and fans lose the ability to regulate continuously. Infrared remote control is the first-choice low-cost solution for realizing the precise control of such appliances. At present, however, some devices with infrared control function still have problems such as single infrared carrier frequency and no infrared encoding library. In addition, since the device is generally installed behind the electrical appliance, the design of the device and the infrared module does not take into account the possibility that the linear propagation of the infrared light may encounter obstacles, thus limiting the flexibility and application scene of the device.

Based on this, this paper integrates digital signal processing and infrared remote control technologies, and considers the actual application requirements, thus designs a split-type smart electricity measurement and control terminal with power data acquisition and analysis, environmental state awareness, and load flexible regulation functions. This intelligent terminal is able to adapt to a complex home environment, which ultimately can lay a solid hardware foundation for realizing effective home energy management and promoting family load participation in demand response.

\section{Hardware system scheme design}

\subsection{Basic hardware structure}

Taking into account the complex changes in the home environment and the characteristics of infrared light linear propagation, the smart electricity monitoring and control terminal developed in this paper adopts a splittype design approach. Specifically, we divide the terminal into two components: the device body and the air conditioning controller. Among them, the device body is used for electrical quantity data collection and circuit on-off control of the electrical equipment, and the infrared controller can be freely placed to avoid the inadequacies of infrared wireless communication and achieve accurate control of the electrical equipment. In this paper, the ZigBee module can be used to achieve wireless communication between the developed device body and the infrared controller.

\subsection{Hardware design of the device body}

The hardware design scheme of the device body is demonstrated in Fig. 1.

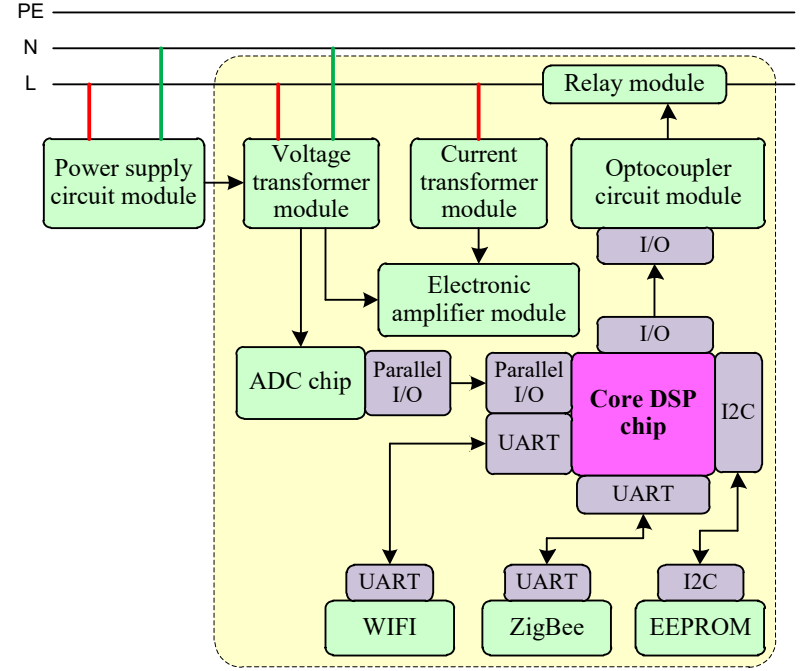

Fig. 1. Hardware framework design of the device body.

\subsubsection{Core processing unit design}

As the smart electricity measurement and control terminal needs to achieve the following functions: including the collection of power frequency electrical quantity, AD conversion and FFT and harmonic analysis. At the same time, it needs to be compatible with both WIFI and ZigBee wireless communication methods. Therefore, the terminal needs to have complex functions, which requires its core processor to have strong computing power, faster control capability, and more communication interfaces. Therefore, in the design of this paper, we chose a DSP chip produced by the United States TI Company, namely TMS320F28335. This chip has a high-speed processing capability of $150 \mathrm{MHz}$ and a 32-bit floating-point processing unit that can carry SCI, SPI, I2C serial communication interfaces, and eCAN, ADC and other functional modules. Therefore, this DSP chip is very suitable as the core processor of the smart power terminal.

\subsubsection{Design of electrical quantities measurement with power frequency}

The idea of processing the electric quantity signals is to convert the electric quantity analog signals through the signal conversion of the voltage/current transformers and the amplifier chip. Thereby, the signals are made to satisfy the $5 \mathrm{~V}$ input amplitude requirement of the ADC chip, and then through the ADC chip, the AD conversion is performed. Finally, these digital signals are transmitted to the DSP chip for digital signal processing. The wiring is illustrated in Fig. 2. 


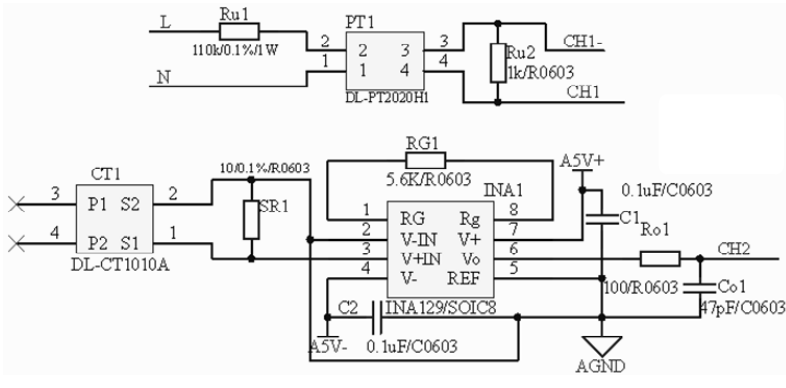

Fig. 2. Design of the voltage and current sampling circuits.

The ADC chip model chosen in this design is AD7606, this chip can realize 16 synchronous sampling, the sampling rate is as high as $200 \mathrm{k}$, can guarantee the sampling speed and precision.

The voltage transformer is a $2 \mathrm{~mA} / 2 \mathrm{~mA}$ current-type voltage transformer with model of DL-PT2020H. The input voltage signal is obtained from the live line and the neutral line. After the current limiting resistor Ru1, the voltage signal is converted into a $2 \mathrm{~mA}$ current signal input to the transformer. In this case, the transformer implements a $1 / 1 \mathrm{AD}$ conversion and outputs $2 \mathrm{~mA}$, and then passes through the parallel-connected resistor $\mathrm{Ru} 2$ at the output to reconvert the current signal into a $2 \mathrm{~V}$ voltage signal to meet the ADC input limit. Current transformer selection is DL-CT1010A, turns ratio is $1000 / 1$. When the input current is a rated value of $16 \mathrm{~A}$, the transformer outputs current of $16 \mathrm{~mA}$, converted into a $320 \mathrm{mV}$ voltage signal through the sampling resistor SR1, and then achieve 10 times the signal amplification through the op amp circuit, and finally achieve $3.2 \mathrm{~V}$ output signal.

Compared with the traditional resistive voltagedividing acquisition method, the influence of the temperature rise of the sampling resistor on the sampling accuracy is avoided, and the accuracy of the transformer sampling is higher. In addition, the transformer realizes electrical isolation between the distribution network side and the device side, thereby protecting the device itself.

\subsubsection{Design of wireless communication function}

The intelligent power terminal supports both ZigBee and WIFI wireless communication methods. The ZigBee communication method is used to issue control commands to the infrared controller, and the data collected by the device body is uploaded to the cloud server through the WIFI communication method.

ZigBee communication power is small, selforganizing ability is strong, communication distance is far, can reach $1 \mathrm{~km}$ as far as possible, the shortcoming is that the transmission speed is slower, it is suitable for the device body and infrared controller this kind of communication that there is less communication volume, communication distance is uncertain. ZigBee module is selected DRF1609, its powerful processing power, easy to use. The intelligent terminal uses a set of serial ports to connect with the ZigBee module, the baud rate is set to 38400 , the device body is set as the master node, the infrared controller is set as the slave node, and the transparent control mode is used to issue the control command.

The WIFI communication speed is fast and the data volume is large. Since a large amount of data needs to be transmitted between the smart terminal and the cloud server, WIFI communication becomes the most suitable method. WIFI module selection for the USR-C215, the module hardware integrated MAC, baseband chip, RF transceiver unit and power amplifier, support WIFI protocol and TCP / IP protocol. The device body is set as a TCP client, and the TCP server is a cloud server for data storage. The transparent transmission mode is used to upload the electrical quantity and environmental quantity information collected in the smart terminal.

\subsubsection{Design of other important circuits}

Each module on the device body requires two different operating voltages of $5 \mathrm{~V}$ or $3.3 \mathrm{~V}$. This design uses the power module HQ05P10 to realize the conversion of $220 \mathrm{~V}$ AC to $5 \mathrm{~V}$ DC. The efficiency of the module is as high as $78 \%$, the output voltage is stable, the error is within $\pm 1 \%$, the ripple noise is low, and the overcurrent protection is provided. Then use TI's TPS73633 regulator chip to achieve $5 \mathrm{~V}$ DC to $3.3 \mathrm{~V}$ DC conversion. The power supply circuit is designed as shown in Fig. 3.
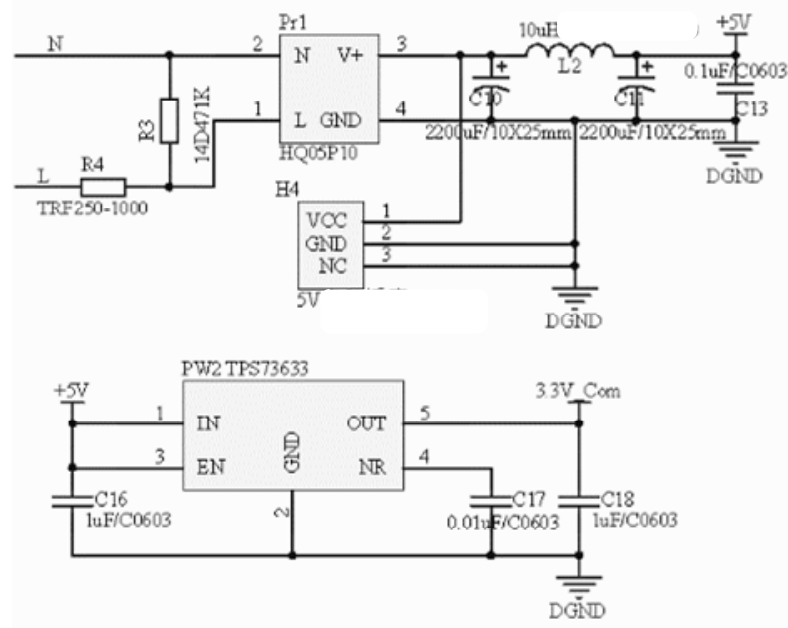

Fig. 3. Design of the power supply circuit.

The device has a relay for controlling the opening and closing of the electrical device. In order to prevent the interference in the circuit breaking process from affecting the normal operation of the device, the design drives the switch on and switch off of the relay through optocoupler isolation, and the circuit connection is shown in Fig. 4. The relay model is HF7520 and the optocoupler chip is PC817. DSP uses an I/O port output high and low to control the relay turn on and turn off. 


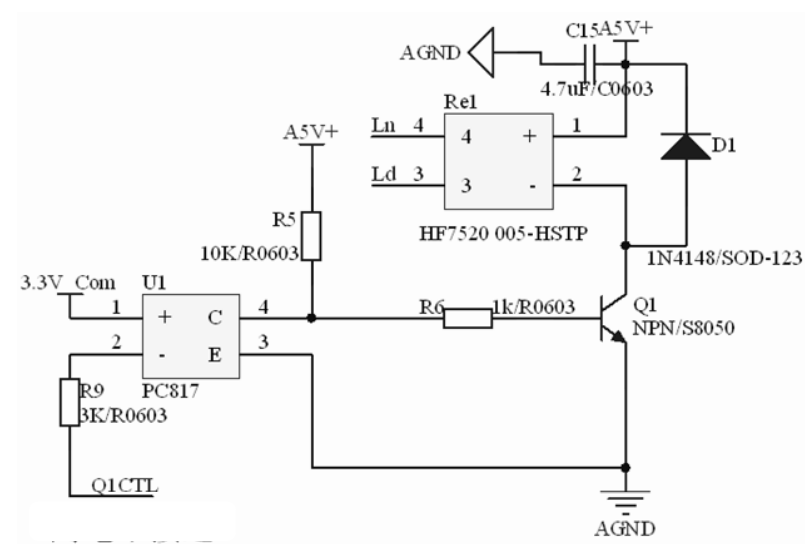

Fig. 4. Design of the optocoupler relay.

In order to avoid the loss of some important information in the device after power down, the device is equipped with a piece of EEPROM chip. The EEPROM is selected as AT24C64AN and I2C communication is used between DSP and EEPROM.

The device is also equipped with a temperature sensor for real-time temperature in the room. The acquisition of temperature data can provide the basis for the decision-making of the control of the airconditioning control of important objects of infrared control, and can also be used for other purposes such as user comfort. The selection of the temperature sensor is AM2302. The sensor is based on the NTC temperature measuring element. The temperature measurement resolution is $0.1^{\circ} \mathrm{C}$ and the measurement error is less than $\pm 0.5^{\circ} \mathrm{C}$.

\subsection{Hardware design of the controller}

\subsubsection{Framework design of the infrared controller}

The overall structure of the infrared controller is shown in Fig. 5. The controller takes the SCM as the core, communicates with the device main body through ZigBee, and controls the infrared control chip according to the command sent by the device main body. The infrared control chip takes the infrared waveform from the code library and sends it to the infrared driving chip to make the infrared transmitting head send out control signals.

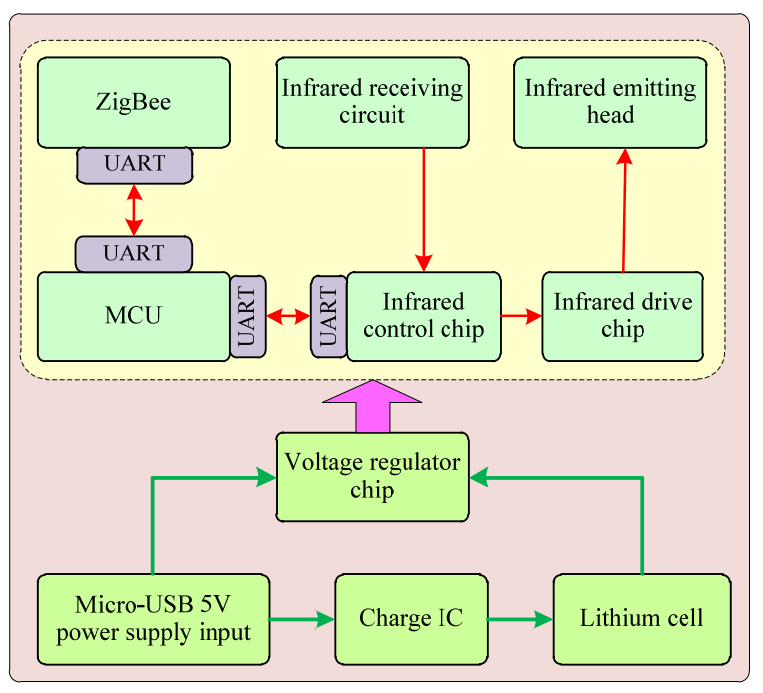

Fig. 5. Framework design of the infrared controller.

\subsubsection{Design of the $M C U$}

In order to realize the interaction logic with the device body and complete the receiving, buffering, parsing, and execution of instructions sent to the device, and controlling the infrared signal of the infrared control chip to send and receive infrared signals and its power-saving state of hibernation, the infrared controller needs a central control unit. Considering the cost and function requirements comprehensively, this device selects the STC15L2K60S2 microcontroller. The STC15L2K60S2 is a single-clock/machine-cycle next-generation 8051 microcontroller produced by STC, which is 8-12 times faster and has 2 sets of ultra-fast asynchronous serial communication ports (UARTs).

A group of UART port of the one-chip computer is connected with ZigBee module, in order to realize and communicate with the apparatus body via the wireless ZigBee communication technology [21-23]; another set of UART port is connected with infrared control chip, used for controlling its movement and working state. Due to the slow sending speed of the infrared instruction, if the device body continuously transmits multiple instructions, the direct transmission to the infrared control chip will cause a conflict. In order to solve this problem, the device implements a circular queue for the instruction cache in the single-chip microcomputer and solves the problem of cooperation between the two high and low speed transmission modes.

\subsubsection{Selection of the infrared control chip}

There are many kinds of electrical products that use the infrared control mode in the electronic market. The difference in manufacturers and models has led to complex and diverse infrared signal coding schemes. In order to enhance the ease of use of the product and avoid the use of infrared coding for lengthy learning configurations before use, the device uses an infrared control chip XK2233 with a rich encoding library to solve this problem. Infrared control chip XK2233 is a high-performance solution for infrared control of 
household appliances, built-in infrared coded information of nearly a thousand types of air conditioner remote control, using carrier learning extension method, can learn infrared signals with different carrier frequencies and arbitrary encoding formats, support for TVs, set-top boxes, DVDs, fans and other home appliances. The chip uses an UART interface to communicate with the outside world. It supports a sleep power-saving mode with a static hold current of less than $6 \mu \mathrm{A}$.

\subsubsection{Design of the infrared receiving circuit}

In order to enhance the expansion capability of the infrared controller, the device realizes the function of receiving and learning the infrared remote control signal. The infrared remote control signal itself is an optical signal and cannot be processed directly by the chip. It is necessary to convert the optical signal into a high-level signal through an infrared reception circuit. The infrared learning circuit is shown as graphically in Fig. 6, which consists of an infrared photodiode and a triode amplifier circuit. This structure is simple and the effect is good.

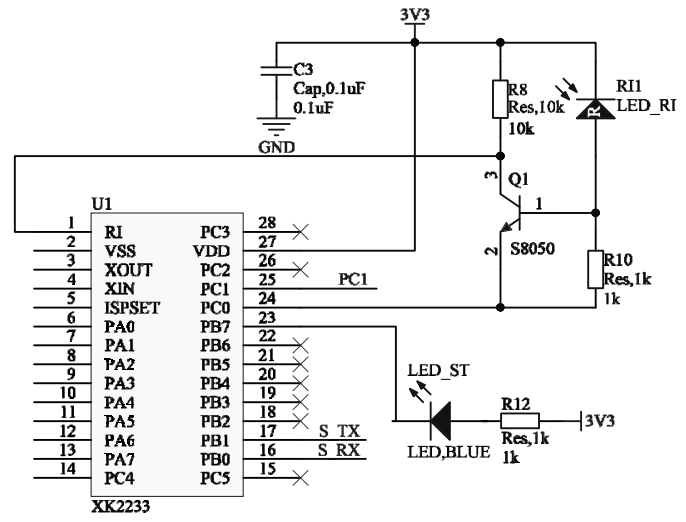

Fig. 6. The principle of the infrared signal learning circuit.

\subsubsection{Design of the infrared transmitting circuit}

Different from the manual alignment of the target device when used with a hand-held remote controller, the main objective of this device is to realize automatic control of unattended electrical appliances. In order to avoid the adjustment of the direction when installing, this device adopts a scheme of radiation distribution of 7 infrared emitters, and increases the pulse intensity to ensure reliable delivery of signals.

The infrared emission circuit consists of a driver chip and seven infrared light emitting diodes. The driver chip is ULN2003A, which is a series of Darlington transistor arrays with high voltage and high current. It features high current gain, high operating voltage, wide temperature range, and strong load capacity. The infrared light emitting diode is a $5 \mathrm{~mm}$ light emitting diode with a light emitting wavelength of $940 \mathrm{~nm}$. Six of the six diodes are distributed on the six vertices of a regular hexagon and emit at an angle of $30^{\circ}$ to the bottom. The other diode is emitted vertically upward at the centre of the hexagon. The principle of the infrared transmitting circuit is shown in Fig. 7.

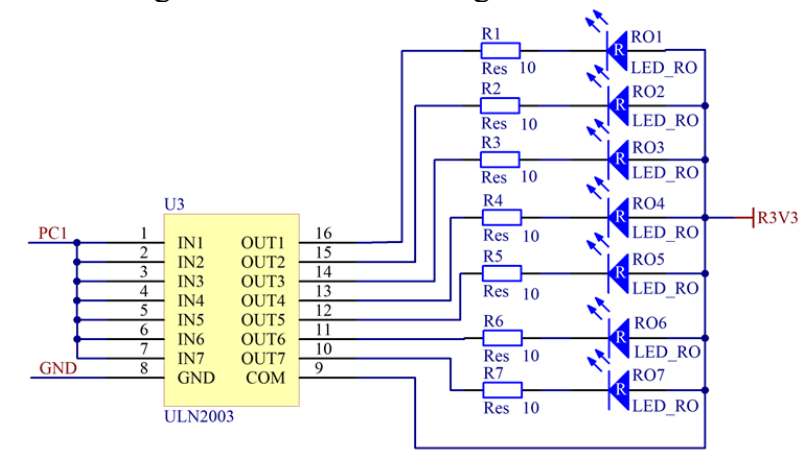

Fig. 7. The principle of the infrared transmitting circuit.

\subsubsection{Design of power supply circuit}

High requirements are put on the flexibility of the installation location of the device, due to the characteristics of the linear propagation of infrared signals, and fundamentally speaking, it imposes requirements on the flexibility of the power supply of the device. In order to solve this problem, the device uses external adapter power supply and built-in rechargeable lithium battery power supply, which greatly reduces the installation cost due to power supply wiring.

The infrared controller power circuit is shown in Fig. 8 . The positive electrode of the two lithium batteries and the positive electrode of the external $5 \mathrm{~V}$ input are each connected to the regulator chip through a Schottky diode with a very low turn-on voltage drop. It can prevent the formation of circulation between the two lithium batteries or lithium battery to external power supply.

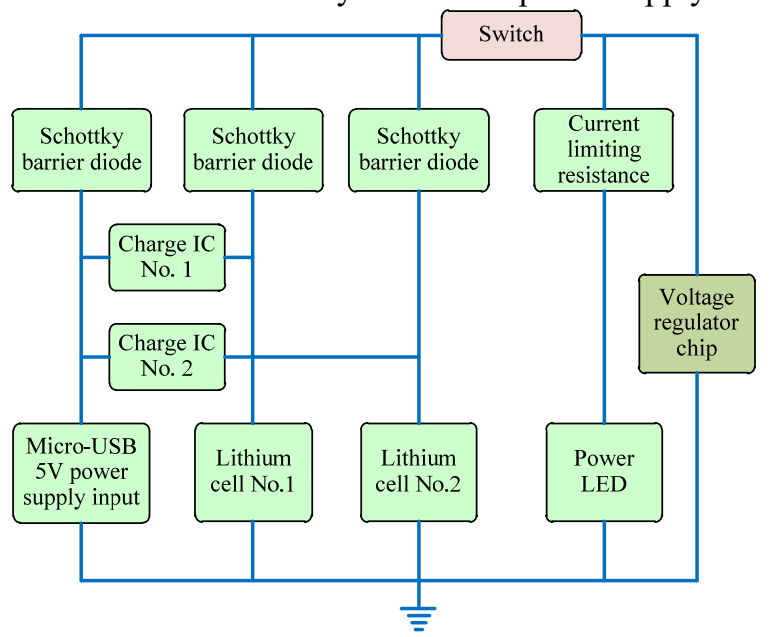

Fig. 8. The principle design of the infrared controller power supply circuit.

The external DC power interface is a Micro-USB interface, which has the characteristics of strong versatility and ease of use. The TP4056 chip used in the charge control chip is easy to use, safe and reliable, and supports a programmable charge current of up to $1000 \mathrm{~mA}$. The lithium battery is a $2600 \mathrm{mAh} 18650$ battery with built-in overcharge and over discharge protection, which greatly improves safety. The XC6206P332MR chip used in the regulator chip is a 
high precision, low power positive voltage regulator with an output voltage rating of $3.3 \mathrm{~V}$, which can be an ideal operating voltage for other devices.

\section{Software programming design}

\subsection{Software programming design of the device body}

The device body program consists of a main program and an interrupt service program, as shown in Fig. 9.

After the power is switched on, the program enters the initialization, configuration communication, timing and interrupt parameters. Then, the program enters a sampling cycle of $1 \mathrm{~s}$ per cycle, and the sampling frequency is 128 sample points per week. After collecting four frequency cycles, data processing is performed to calculate the voltage and current RMS values, power, power factor, harmonic content, and other electrical parameters through discrete sample points. Then store these data in EEPROM to avoid data loss after power-down. Finally wait for the end of the $1 \mathrm{~s}$ timer to restart sampling and repeat the above process. The interrupt service routine triggers activation after the device body receives the cloud command, first determining whether it is the local address, and if so, determining the command: if the command is data upload, the electrical quantity information stored in the device is uploaded via WIFI. If it is an IR-controlled

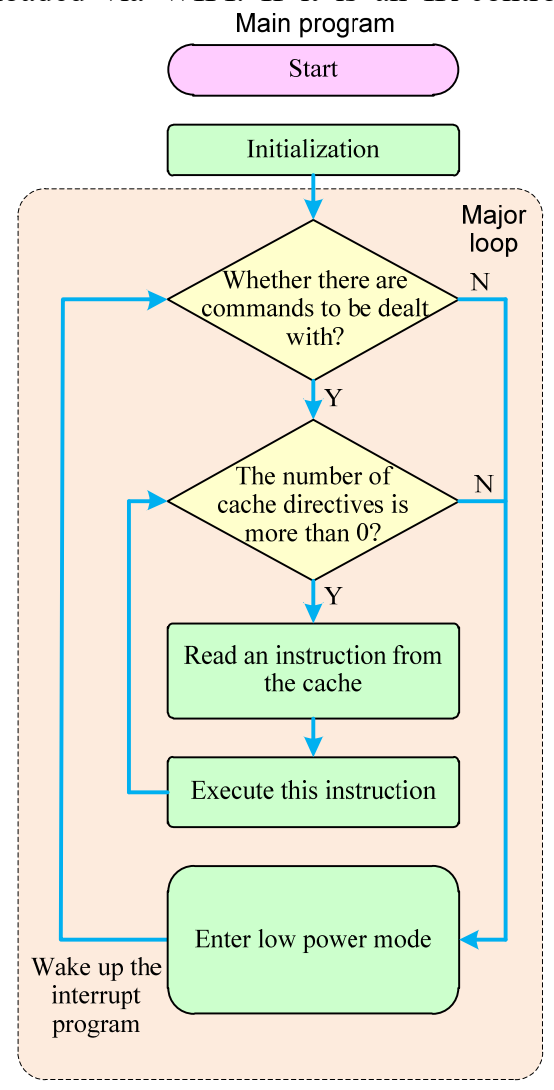

Fig. 10. Design of the main program and the interrupt service routine in the infrared controller.

The main program completes the hardware and software initialization of the device, and then enters the main loop to process the received instructions. When

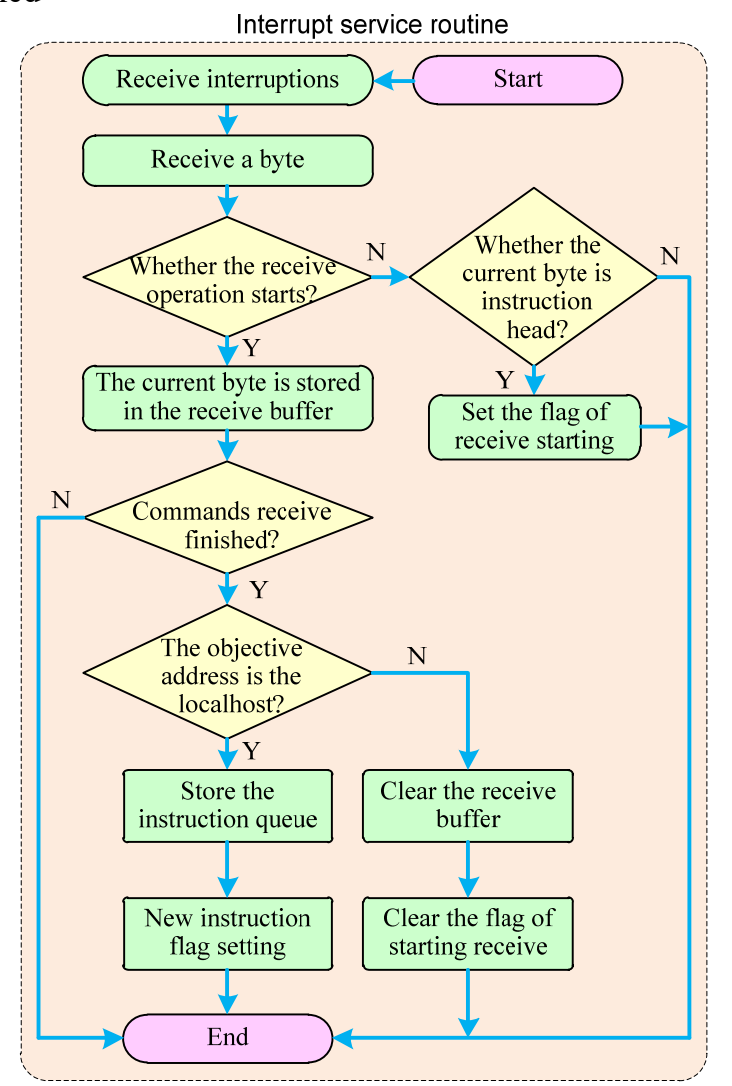

command, the ZigBee forwarding command allows the IR controller to respond and execute.
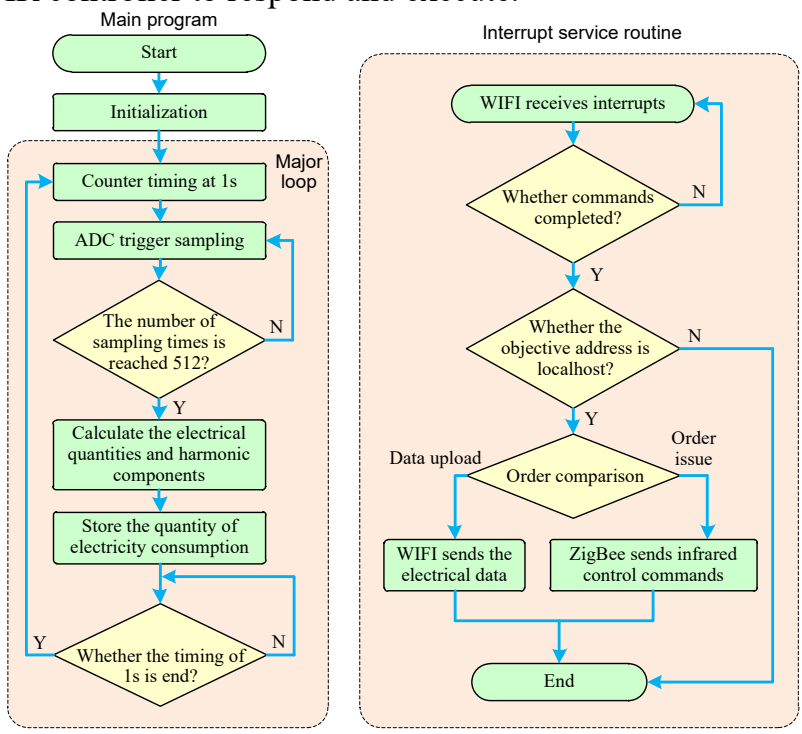

Fig. 9. Design of the main program and the interrupt service routine in the device body.

\subsection{Software programming design of the infrared controller}

The infrared controller program is mainly composed of a main program and an interrupt service program, as shown in Fig. 10.

\section{Receive a byte}

finished?

The objective

ddress is the

$\checkmark \mathrm{Y}$

there are no pending instructions, the entire device enters a low-power state and waits for an interrupt to wake up to achieve power saving. The interrupt service routine is 
used to respond to the UART receive interrupt, cache the received byte to form a complete instruction, determine whether the target of the instruction is native, and store the instruction in the instruction queue, otherwise the instruction is discarded.

Based on the previous hardware design scheme, the split-type smart power measurement and control terminal based on DSP and infrared remote control developed in this paper is shown in Fig. 11 and Fig. 12 below.

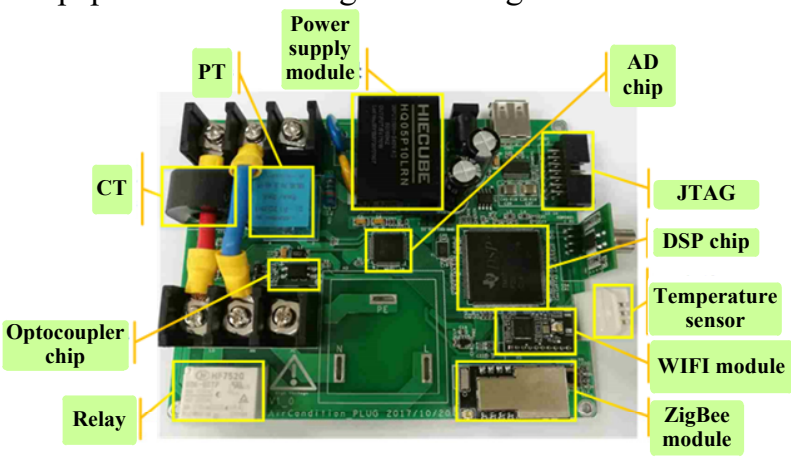

Fig. 11. Prototype of the device body developed in this paper.

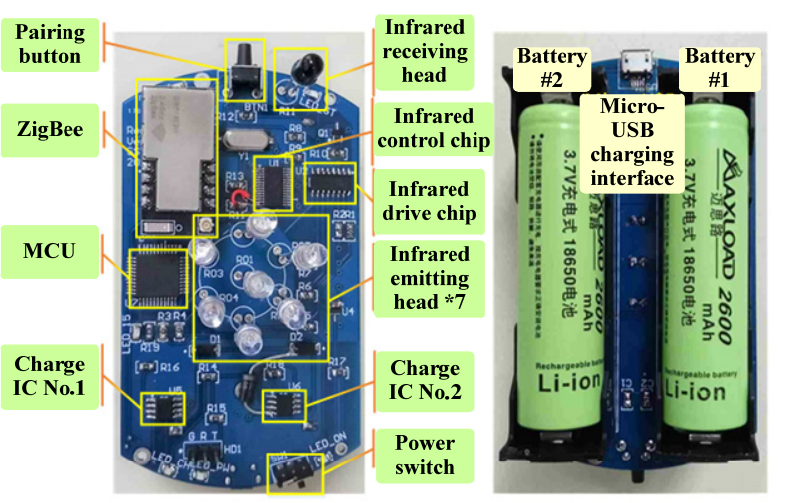

Fig. 12. Prototype of the infrared controller developed in this paper.

\section{Experimental verification}

\subsection{Testing of the device body}

In order to verify the power information collection capability of the device body, we use the electrical measuring instrument KS833 integrated calibration device as a standard source to test the performance of the device. The results are presented as follows. Fig. 13(a), (b), and (c) show the testing result of voltage, current, and power factor, respectively. We can see from the testing results shown in Fig. 13 that the relative errors of voltage, current, and power factor in three groups of testing are all smaller than $1 \%$, which verifies that the developed device body possesses good measurement accuracy.

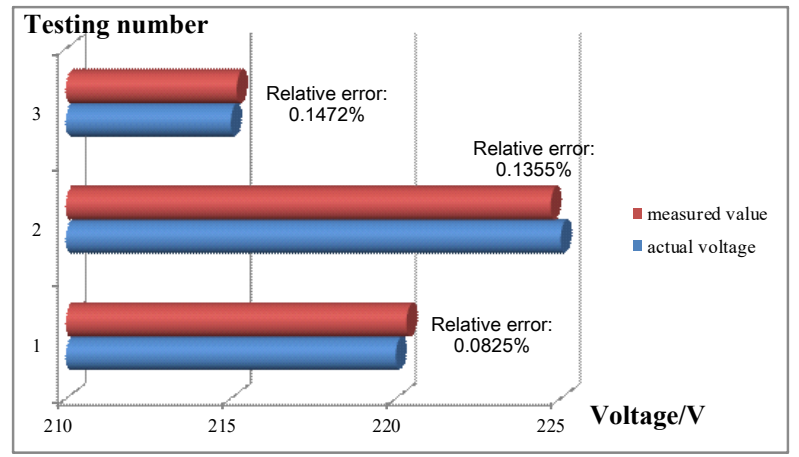

(a) Voltage testing results

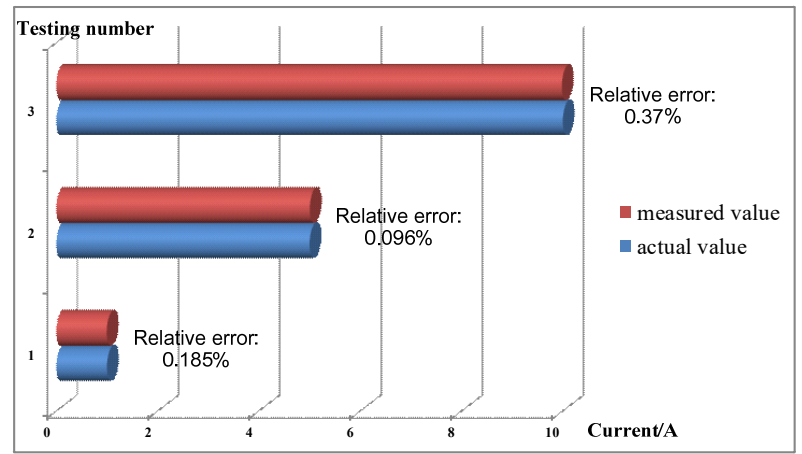

(b) Current testing results

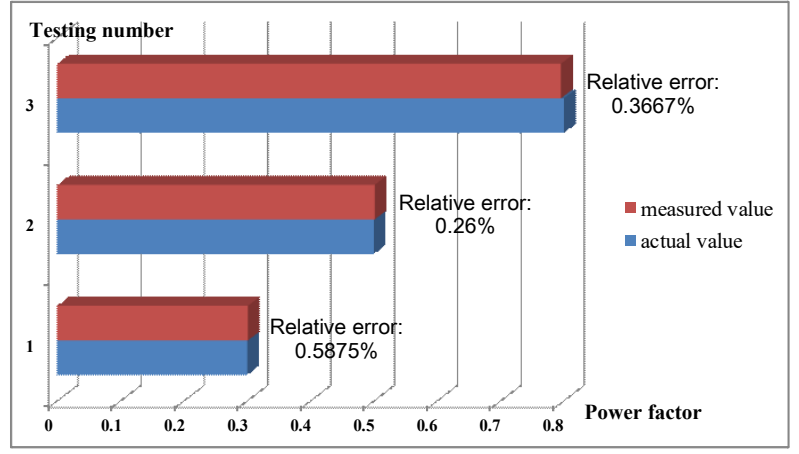

(c) Power factor testing results

Fig. 13. Testing results.

\subsection{Testing of the infrared controller}

For the infrared controller, we describe the position of the controlled target electrical appliance relative to the controller by establishing a spherical coordinate system on the device. The origin of the coordinate system is the centre of the centre of the infrared diode. The $z$-axis is perpendicular to the horizontal. The $x$-axis points to the centre of the infrared diode closest to the ZigBee module. The $y$-axis points 90 degrees to the left of the axis. In this coordinate system, the emission directions of the six infrared LEDs on the circumference can be expressed as $\theta=60^{\circ}$ and $\varphi=k \times 60^{\circ}(k=0,1, \ldots, 5)$, and the centre infrared LED is represented as $\theta=0^{\circ}$.

In order to test the coverage ability of the infrared controller's transmitted signal, the transmission angle and transmission distance were tested. The experimental results are shown in the Table 1, where the distance $r$ is taken $2 \mathrm{~m}$. The testing results of the infrared emitting distance are shown in Table 2. 
Table 1. The testing results of the infrared emitting angle.

\begin{tabular}{cccl}
\hline distance $r / \mathrm{m}$ & $\varphi /$ degree & $\theta /$ degree & results \\
\hline 0 & 0 & succeed \\
0 & 30 & succeed \\
0 & 60 & succeed \\
2 & 0 & 90 & succeed \\
\cline { 2 - 4 } & 30 & 0 & succeed \\
& 30 & 30 & succeed \\
& 30 & 60 & succeed \\
& 30 & 90 & succeed \\
\hline
\end{tabular}

Table 2. The testing results of the infrared emitting distance.

\begin{tabular}{cccc}
\hline distance $r / \mathrm{m}$ & $\varphi /$ degree & $\theta /$ degree & results \\
\hline 3 & 30 & 30 & succeed \\
4 & 30 & 30 & succeed \\
5 & 30 & 30 & succeed \\
6 & 30 & 30 & succeed \\
7 & 30 & 90 & failed \\
7 & 0 & 60 & succeed \\
\hline
\end{tabular}

\section{Conclusion}

This paper has developed a novel split-type intelligent electricity consumption monitoring and control terminal with digital signal processing and infrared precision control capabilities, which is able to effectively overcome the lack of flexibility in intelligent power terminal control methods and weak data acquisition and processing capabilities. This developed terminal can realize accurate collection and processing of power consumption information, as well as accurate infrared control functions, such that we can solve the issue of traditional non-stop-on-off control mode to make the adjustable power equipment lose the ability of continuous regulation. Therefore, this smart terminal is capable of changing the situations where previous infrared controllers possess single carrier frequency and have no infrared encoding library.

Thanks to the split-type and rechargeable design, the terminal developed in this paper is easy to install and adapt to a complex home environment. The split-type intelligent measurement and control terminal designed in this paper will have the following use in the future. One is to lay a solid foundation of hardware for the theoretical application of the upper layer system; second is to enable many more comprehensive and diverse control strategies to be put into practice; and the last one is to provide favorable conditions for the next step of investigation, including the research on effective household energy management in the context of energy Internet, and promoting household loads participating in demand response.

\section{Acknowledgement}

The work is partially supported by the Key Science and Technology Projects of China Southern Power Grid (Grant No. GZKJQQ00000419).

\section{References}

1. L.F. Cheng, T. Yu, X.S. Zhang, et al. Cyberphysical-social systems based smart energy robotic dispatcher and its knowledge automation: framework, techniques and challenges. Proceedings of the CSEE, 2018, 38(1): 25-40.

2. A.Q. Huang, M.L. Crow, G.T. Heydt, et al. The Future Renewable Electric Energy Delivery and Management (FREEDM) System: the energy internet. Proceedings of the IEEE, 2010, 99(1):133148.

3. L.H. Tsoukalas, R. Gao. From smart grids to an energy internet: Assumptions, architectures and requirements. In Proceedings of the International Conference on Electric Utility Deregulation and Restructuring and Power Technologies. IEEE, 2008: 94-98.

4. L.F. Cheng, T. Yu. Nash equilibrium-based asymptotic stability analysis of multi-group asymmetric evolutionary games in typical scenario of electricity market. IEEE Access, accepted, DOI: 10.1109/ACCESS.2018.2842469.

5. L.F. Cheng, B.X. Chen, T. Yu. Study the influence of the implementation of demand side management on generation system reliability. Applied Mechanics and Materials, 2014, 971-973: 2004-2012.

6. M. Zhong, W.M. Lai. Research and practice of demand side response at home and abroad. Guizhou Electric Power Technology. 2016, 19(10): 21-24.

7. L.F. Cheng, B. Zhou, T. Yu. Design and develop resources investigation and evaluation system of electric power DSM. Applied Mechanics and Materials, 2014, 986-987: 271-278.

8. M. Zeng, X. Han, J.H. Sun, et al. Key issues and prospects of automated demand response under energy internet background. Electric Power Construction, 2017, 38(2): 21-27.

9. M. Yang, Y.C. Wang, D. Xiang, et al. Model and optimization analysis for household electricity consumption system in smart grid. Power Demand Side Management. 2017, 19(4): 6-10.

10. L.F. Cheng, Z.Y. Zhang, H.R. Jiang, et al. Local energy management and optimization: A novel energy universal service bus system based on energy Internet technologies. Energies, 2018, 11(5), 1160. DOI: $10.3390 /$ en 11051160

11. W. Wang, G.Y. He, J.L. Wan, et al. Model and optimization analysis for household electricity consumption system in smart grid. Power Demand Side Management, 2017, 19(4): 6-10. 
12. L.H. Zhang, S.T. Wang, Z.L. Yi, et al. The design and implementation of family comprehensive energy management system facing the smart power. Electrical Measurement \& Instrumentation, 2010, 9: 35-38.

13. D.S. Wu, Z.W. Li, D. Meng, et al. Design of intelligent interactive terminal of household intelligent power management system. Automation \& Instrumentation, 2017, 1: 34-38+42.

14. K.H. Wen, H.D. Huang, W.H. Li, et al. The typical design and application of intelligent measurement system based on cloud platform. Electrical Measurement \& Instrumentation, 2016, 53(5):118123.

15. C.Y. Fu, G. Ma, M.M. Zhou. Design of advanced metering infrastructure based on internet of things. Electrical Measurement \& Instrumentation, 2012, 49(7): 45-48.

16. L.F. Cheng, M.X. Xu, T. Yu, et al. A kind of smart home electricity controller based on active distribution networks and ATT7035 chip. In proceedings of the 2015 4th International Conference on Measurement, Instrumentation and Automation (ICMIA 2015), Shenzhen China, August 1-2, 2015, pp. 813-819.

17. M.X. Shen, Y. Sun, B. Li. Study on the architecture and application of the automatic demand response system based on the technology of IOT. Electrical Measurement \& Instrumentation. 2014, 51(24): 96100.

18. D.D. Li, L.L. Cui, S.F. Lin, et al. Study of smart power utilization system and development of smart controller for homes. Power System Protection and Control. 2013, 41(4): 123-129.

19. L.Y. Liangli. Design of STM32-based smart socket system with wireless infrared remote control function. Modern Electronics Technique. 2015, 38(19): 156-159.

20. S.F. Liu. The research and design of power demand response controller for residential customers. Master Thesis, North China Electric Power University, Beijing, China, 2016.

21. W. Wang, G. He, J. Wan. Research on Zigbee wireless communication technology. In Proceedings of the International Conference on Electrical and Control Engineering. IEEE, 2011:1245-1249.

22. P. Tao, X. Liu. Hybrid wireless communication system using zigbee and WiFi technology in the coalmine tunnels. In Proceedings of the Third International Conference on Measuring Technology and Mechatronics Automation. IEEE Computer Society, 2011: 340-343.

23. Z. Qi, Y. Zhang, L. Zhao, et al. A new wireless communication system based on ZigBee technology. In Proceedings of the International Conference on Photonics and Image in Agriculture Engineering. International Society for Optics and Photonics, 2009: 96. 